\title{
Neutrino charge radii from COHERENT elastic neutrino-nucleus scattering
}

\author{
M. Cadeddu* \\ Dipartimento di Fisica, Università degli Studi di Cagliari, \\ and INFN, Sezione di Cagliari, Complesso Universitario di Monserrato-S.P. \\ per Sestu Km 0.700, 09042 Monserrato (Cagliari), Italy \\ C. Giunti ${ }^{\dagger}$ \\ Istituto Nazionale di Fisica Nucleare (INFN), Sezione di Torino, Via P. Giuria 1, I-10125 Torino, Italy \\ K. A. Kouzakov \\ Department of Nuclear Physics and Quantum Theory of Collisions, Faculty of Physics, \\ Lomonosov Moscow State University, Moscow 119991, Russia \\ Y. F. $\mathrm{Li}^{\S}$ \\ Institute of High Energy Physics, Chinese Academy of Sciences, Beijing 100049, China \\ and School of Physical Sciences, University of Chinese Academy of Sciences, \\ Beijing 100049, China \\ A. I. Studenikin \\ Department of Theoretical Physics, Faculty of Physics, \\ Lomonosov Moscow State University, Moscow 119991, Russia \\ and Joint Institute for Nuclear Research, Dubna 141980, Moscow Region, Russia \\ Y. Y. Zhang \\ Institute of High Energy Physics, Chinese Academy of Sciences, Beijing 100049, China \\ and School of Physical Sciences, University of Chinese Academy of Sciences, \\ Beijing 100049, China
}

(Received 15 October 2018; published 26 December 2018)

\begin{abstract}
Coherent elastic neutrino-nucleus scattering is a powerful probe of neutrino properties, in particular of the neutrino charge radii. We present the bounds on the neutrino charge radii obtained from the analysis of the data of the COHERENT experiment. We show that the time information of the COHERENT data allows us to restrict the allowed ranges of the neutrino charge radii, especially that of $\nu_{\mu}$. We also obtained for the first time bounds on the neutrino transition charge radii, which are quantities beyond the standard model.
\end{abstract}

DOI: 10.1103/PhysRevD.98.113010

\section{INTRODUCTION}

Neutrinos are widely believed to be neutral particles, but in reality they could have a very small electrical charge and it is very likely that they have charge radii (see the review in

\footnotetext{
*matteo.cadeddu@ca.infn.it

†carlo.giunti@to.infn.it

*kouzakov@gmail.com

\$liyufeng@ihep.ac.cn

"studenik@srd.sinp.msu.ru

'zhangyiyu@ihep.ac.cn
}

Published by the American Physical Society under the terms of the Creative Commons Attribution 4.0 International license. Further distribution of this work must maintain attribution to the author(s) and the published article's title, journal citation, and DOI. Funded by SCOAP.
Ref. [1]). Indeed, in the standard model neutrinos have charge radii of the order of $10^{-33} \mathrm{~cm}^{2}$ [2-13]. In this paper we consider the effects of the neutrino charge radii on coherent elastic neutrino-nucleus scattering [14-17] and we present the results on the values of the neutrino charge radii obtained from the analysis of the data of the COHERENT experiment $[18,19]$.

Coherent elastic neutrino-nucleus scattering is a process predicted a long time ago [20-22], which was observed for the first time in 2017 in the COHERENT experiment $[18,19]$. The difficulty is that it is necessary to observe nuclear recoils with very small kinetic energy $T$, smaller than a few $\mathrm{keV}$, in order to satisfy the coherence requirement $|\vec{q}| R \ll 1$ [23], where $|\vec{q}| \simeq \sqrt{2 M T}$ is the threemomentum transfer, $R$ is the nuclear radius of a few fm, and $M$ is the nuclear mass, of the order of $100 \mathrm{GeV}$ for 
TABLE I. Experimental limits for the neutrino charge radii.

\begin{tabular}{|c|c|c|c|c|}
\hline Process & Collaboration & Limit $\left[10^{-32} \mathrm{~cm}^{2}\right]$ & $\mathrm{CL}$ & Ref. \\
\hline Reactor $\bar{\nu}_{e}-e$ & $\begin{array}{l}\text { Krasnoyarsk } \\
\text { TEXONO }\end{array}$ & $\begin{array}{c}\left|\left\langle r_{\nu_{e}}^{2}\right\rangle\right|<7.3 \\
-4.2<\left\langle r_{\nu_{e}}^{2}\right\rangle<6.6\end{array}$ & $\begin{array}{l}90 \% \\
90 \%\end{array}$ & $\begin{array}{l}{[43]} \\
{[44]^{\mathrm{a}}}\end{array}$ \\
\hline Accelerator $\nu_{e}-e$ & $\begin{array}{l}\text { LAMPF } \\
\text { LSND }\end{array}$ & $\begin{array}{c}-7.12<\left\langle r_{\nu_{\nu}}^{2}\right\rangle<10.88 \\
-5.94<\left\langle r_{\nu_{\rho}}^{2}\right\rangle<8.28\end{array}$ & $\begin{array}{l}90 \% \\
90 \%\end{array}$ & $\begin{array}{l}{[45]^{\mathrm{a}}} \\
{[46]^{\mathrm{a}}}\end{array}$ \\
\hline Accelerator $\nu_{\mu}-e$ and $\bar{\nu}_{\mu}-e$ & $\begin{array}{l}\text { BNL-E734 } \\
\text { CHARM-II }\end{array}$ & $\begin{array}{c}-5.7<\left\langle r_{\nu_{\mu}}^{2}\right\rangle<1.1 \\
\left|\left\langle r_{\nu_{\mu}}^{2}\right\rangle\right|<1.2\end{array}$ & $\begin{array}{l}90 \% \\
90 \%\end{array}$ & $\begin{array}{l}{[47]^{\mathrm{a}, \mathrm{b}}} \\
{[48]^{\mathrm{a}}}\end{array}$ \\
\hline
\end{tabular}

${ }^{\mathrm{a}}$ Corrected by a factor of two due to a different convention.

${ }^{\mathrm{b}}$ Corrected in Ref. [49].

heavy nuclei. The observation of coherent elastic neutrinonucleus scattering opens up a new and powerful way to probe the properties of nuclei, neutrinos, weak interactions, and new physics beyond the standard model [24-32]. Indeed, the first measurements of the COHERENT experiment have already produced interesting results for nuclear physics [33], neutrino properties and interactions [17,34-37], and weak interactions [38].

The problem of correctly defining the neutrino charge radius in the context of the standard model and beyond has a long history (see the review in Ref. [1]). The authors of one of the first studies [39] found that in the standard model and in the unitary gauge the neutrino charge radius is ultraviolet divergent and, hence, not a physical quantity. A direct oneloop calculation $[40,41]$ of the neutrino charge radius, accounting for contributions of a complete set of proper vertexes and $\gamma-Z$ self-energy Feynman diagrams, performed in a general $R_{\xi}$ gauge for a massive Dirac neutrino, also gave a divergent result. The solution to the problem of obtaining a charge radius that is gauge-independent, finite and independent of the external probe (see Ref. [7] for a detailed discussion) is achieved by including appropriate additional diagrams in the calculation of the neutrino electromagnetic form factor. In the usual approach, the Feynman diagrams are treated individually, and each diagram either contributes to the form factor in its entirety or it does not contribute at all. However, this method yields an infinite and gauge-dependent charge radius. The problem is that certain diagrams, which at first glance do not appear to be relevant for the calculation of the form factor, contain pieces that cannot be distinguished from the contributions of the regular diagrams and must therefore be included in order to obtain a finite result. The appropriate way to include those diagrams, found in Ref. [7], is based on the pinch technique. The resulting neutrino charge radii are finite and independent of the gauge and the external probe [8-10].

Until now, the neutrino charge radii have been typically searched in elastic neutrino-electron scattering experiments. Summaries of the limits obtained so far in this way can be found in Refs. [1,42] and in Table I of this paper. For small energy transfer $T$, both the standard model cross section and the effect of the neutrino charge radii in the case of elastic neutrino-electron scattering turn out to be smaller by a factor of the order of $M / m_{e}$ with respect to the case of coherent elastic neutrino-nucleus scattering. Therefore, in terms of data collection, coherent elastic neutrino-nucleus scattering experiments have a greater potential for investigating the neutrino charge radii than the measurements of neutrino-electron scattering.

In this paper we calculate accurately the limits on the neutrino charge radii from the analysis of the COHERENT data, starting with a discussion of the theoretical framework in Sec. II, which includes also a summary of the previous experimental limits in Table I and a discussion of other limits obtained with combined analyses of the data of different experiments. In Secs. III and IV we present, respectively, the results obtained from the analyses of the time-integrated COHERENT energy spectrum and the time-dependent COHERENT data. In Sec. V we draw our conclusions.

\section{THEORETICAL FRAMEWORK}

In the fundamental theory of electromagnetic neutrino interactions the neutrino charge radii are defined for the massive neutrinos (see the review in Ref. [1]). However, the effects of neutrino oscillations can be neglected for experiments with a short distance between the neutrino source and detector, as in the setup of the COHERENT experiment. In this case one can consider the effective charge radius $\left\langle r_{\nu_{\ell}}^{2}\right\rangle$ of a flavor neutrino $\nu_{\ell}$, with $\ell=e, \mu, \tau$. Since in the ultrarelativistic limit the charge form factor conserves the neutrino helicity, as the standard model weak interactions, the contribution of $\left\langle r_{\nu_{\ell}}^{2}\right\rangle$ to the elastic scattering of $\nu_{\ell}$ with a charged particle adds coherently to the standard model weak interactions and can be expressed through the shift $[6,50,51]$

$$
\sin ^{2} \vartheta_{W} \rightarrow \sin ^{2} \vartheta_{W}\left(1+\frac{1}{3} m_{W}^{2}\left\langle r_{\nu_{\ell}}^{2}\right\rangle\right)
$$

where $\vartheta_{W}$ is the weak mixing angle, $m_{W}$ is the mass of the $W$ boson, and $\ell=e, \mu, \tau$. This shift follows from the expression $^{1}$

\footnotetext{
${ }^{1}$ For simplicity we omitted a term $q_{\mu} \phi / q^{2}$ whose contribution vanishes in the coupling with a conserved current as in neutrinoelectron and neutrino-nucleon scatterings.
} 


$$
\Lambda_{\mu}^{(\nu)}(q)=\gamma_{\mu} F_{\nu}\left(q^{2}\right) \simeq \gamma_{\mu} q^{2} \frac{\left\langle r^{2}\right\rangle}{6}
$$

of the effective electromagnetic interaction vertex of neutrinos in the standard model, where $F_{\nu}\left(q^{2}\right)$ is a form factor, $q$ is the four-momentum transfer and the approximation holds for small values of $q^{2}$. The charge radius is given by (see Ref. [1])

$$
\left\langle r^{2}\right\rangle=\left.6 \frac{d F_{\nu}\left(q^{2}\right)}{d q^{2}}\right|_{q^{2}=0} .
$$

Unfortunately in the literature there is some confusion on the size and the sign of the shift of $\sin ^{2} \vartheta_{W}$ due to a neutrino charge radius. The authors of Ref. [49] considered a shift that has the same magnitude but opposite sign. The authors of Refs. [17,52] considered a shift that is twice as large, with the same sign, which corresponds to values of the neutrino charge radii which are half of ours. The authors of Ref. [15,53] considered a shift that is twice as large, with opposite sign. This implies that the standard model values of the neutrino charge radii reported in Ref. [15], according to the calculations in Refs. [8-10], are half and with opposite sign with respect to those that would be obtained in our framework. We think that the sign of the shift can be considered as a convention on the definition of $\left\langle r^{2}\right\rangle$ as $\pm 6 d F_{\nu}\left(q^{2}\right) /\left.d q^{2}\right|_{q^{2}=0}$. Indeed, in Ref. [8] it is explicitly written that $\left\langle r^{2}\right\rangle=-6 d F_{\nu}\left(q^{2}\right) /\left.d q^{2}\right|_{q^{2}=0}$, which differs by a sign from our definition in Eq. (3). We think that the difference of a factor of two is due to the assumption of a contribution to the effective electromagnetic interaction vertex of an anapole moment with the same value of the charge radius, which leads to a doubling of the shift of $\sin ^{2} \vartheta_{W}$. This is indicated by Eq. (8) ${ }^{2}$ of Ref. [10], where the $\gamma^{5}$ term is due to an anapole moment assumed to have the same value as the charge radius [9]. Acting on left-handed spinors with $1-\gamma^{5}=2$ leads to the doubling of the shift of $\sin ^{2} \vartheta_{W}$. However, as explained in Ref. [1] this approach is not well justified because in the standard model there is only the form factor in Eq. (2), which can be interpreted either as a charge radius or as an anapole moment. ${ }^{3}$ Taking into account these considerations, in our framework the standard model predictions of the neutrino charge radii calculated in Refs. [8-10] are given by

$$
\left\langle r_{\nu_{\ell}}^{2}\right\rangle_{\mathrm{SM}}=-\frac{G_{\mathrm{F}}}{2 \sqrt{2} \pi^{2}}\left[3-2 \ln \left(\frac{m_{\ell}^{2}}{m_{W}^{2}}\right)\right],
$$

where $m_{W}$ and $m_{\ell}$ are the $W$ boson and charged lepton masses $(\ell=e, \mu, \tau)$. The shift of $\sin ^{2} \vartheta_{W}$ given by this

\footnotetext{
${ }^{2}$ Eq. (2.8) in the arXiv version.

${ }^{3}$ Since the anapole moments have the same effects on the interactions of ultrarelativistic neutrinos as the corresponding charge radii, the phenomenological constraints on the charge radii apply also to the anapole moments (multiplied by -6 in the conventions of Ref. [1]).
}

expression of $\left\langle r_{\nu_{\ell}}^{2}\right\rangle_{\mathrm{SM}}$ and Eq. (1) is in agreement with the main contribution calculated in Refs. $[14,54]$ and with the shift given in Ref. [55]. Numerically, we have

$$
\begin{aligned}
& \left\langle r_{\nu_{e}}^{2}\right\rangle_{\mathrm{SM}}=-0.83 \times 10^{-32} \mathrm{~cm}^{2}, \\
& \left\langle r_{\nu_{\mu}}^{2}\right\rangle_{\mathrm{SM}}=-0.48 \times 10^{-32} \mathrm{~cm}^{2}, \\
& \left\langle r_{\nu_{\tau}}^{2}\right\rangle_{\mathrm{SM}}=-0.30 \times 10^{-32} \mathrm{~cm}^{2} .
\end{aligned}
$$

The current experimental bounds on $\left\langle r_{\nu_{e}}^{2}\right\rangle$ and $\left\langle r_{\nu_{\mu}}^{2}\right\rangle$ are listed in Table I, which is a corrected version of Table V of Ref. [1].

The global fit of low-energy $\nu_{e}-e$ and $\bar{\nu}_{e}-e$ measurements presented in Ref. [52] yielded the $90 \%$ CL allowed interval

$$
-0.26 \times 10^{-32}<\left\langle r_{\nu_{e}}^{2}\right\rangle<6.64 \times 10^{-32} \mathrm{~cm}^{2},
$$

where we have rescaled Eq. (8) of Ref. [52] taking into account the factor of two difference in the definition of the charge radii. This range excludes the standard model value of $\left\langle r_{\nu_{e}}^{2}\right\rangle$ in Eq. (5). However, we think that the allowed range in Eq. (8) must be corrected, because it has been obtained assuming the on-shell standard model value $\sin ^{2} \vartheta_{W}^{\text {on-shell }}=0.2227 \pm 0.0004$ obtained from a fit of high-energy electroweak measurements that do not involve neutrino-nucleon scattering [56] and considering the $\nu_{e}-e$ coupling $g_{V}^{\nu_{e} e}=\frac{1}{2}+2 \sin ^{2} \vartheta_{W}^{\text {on-shell }}=0.9454 \pm 0.0008$. At low energies the effective $\nu_{e}-e$ coupling is given by [57]

$$
g_{V}^{\nu_{e} e}=1+\rho_{\nu e}\left(-\frac{1}{2}+2 \hat{\kappa}_{\nu e} \hat{s}_{Z}^{2}\right),
$$

where $\rho_{\nu e}=1.0126 \pm 0.0016, \hat{\kappa}_{\nu e}=0.9791 \pm 0.0025$, and $\hat{s}_{Z}^{2}$ is the value of $\sin ^{2} \vartheta_{W}$ at the $Z$ pole in the $\overline{\mathrm{MS}}$ renormalization scheme. From the LEP measurements at the $Z$ pole, which do not involve neutrino-nucleon scattering, $\sin ^{2} \vartheta_{W}^{\text {on-shell }}=0.22331 \pm 0.00062$ [58]. The corresponding value of $\hat{s}_{Z}^{2}$ is given by $\hat{s}_{Z}^{2}=(1.0348 \pm 0.0002) \sin ^{2} \vartheta_{W}^{\text {on-shell }}$ [42], which leads to $\hat{s}_{Z}^{2}=0.2311 \pm 0.0006$ and, using Eq. (9), $g_{V}^{\nu_{e} e}=0.952 \pm 0.002$. Hence, the limits in Eq. (8) of Ref. [52] must be shifted by $-(0.14 \pm 0.04) \times 10^{-32} \mathrm{~cm}^{2}$. Adding in quadrature the uncertainties, we obtained the 90\% CL allowed interval

$$
-0.54 \times 10^{-32}<\left\langle r_{\nu_{e}}^{2}\right\rangle<6.37 \times 10^{-32} \mathrm{~cm}^{2} .
$$

This allowed interval still excludes the standard model value of $\left\langle r_{\nu_{e}}^{2}\right\rangle$ in Eq. (5), but less strongly than the interval in Eq. (8). We think that this tension requires further investigations, that will be carried out elsewhere.

Constraints on $\left\langle r_{\nu_{\mu}}^{2}\right\rangle$ have been obtained in Ref. [49] from a reanalysis of the CCFR [59] and CHARM-II [48] data on $\nu_{\mu}-e$ and $\bar{\nu}_{\mu}-e$ scattering. Taking into account the sign difference in the definition of the charge radii, in our framework the $90 \%$ allowed interval in Eq. (4.7) of Ref. [49] becomes 


$$
-0.68 \times 10^{-32}<\left\langle r_{\nu_{\mu}}^{2}\right\rangle<0.52 \times 10^{-32} \mathrm{~cm}^{2} .
$$

The standard model value of $\left\langle r_{\nu_{\mu}}^{2}\right\rangle$ in Eq. (6) is within this interval. The closeness of its value to the lower limit indicates that future experiments may be able to measure $\left\langle r_{\nu_{\mu}}^{2}\right\rangle$.

The prescription in Eq. (1) takes into account the contributions to neutrino interactions of the charge radii of the three flavor neutrinos $\nu_{e}, \nu_{\mu}, \nu_{\tau}$. These are the only charge radii that exist in the standard model, because the generation lepton numbers are conserved. However, in theories beyond the standard model neutrinos can have transition charge radii $\left\langle r_{\nu_{\ell \ell^{\prime}}}^{2}\right\rangle$ that change the neutrino flavor. For example, in massive neutrino theories the charge radii are defined in the mass basis of the physically propagating neutrinos. The charge radii $\left\langle r_{\nu_{\ell \ell^{\prime}}}^{2}\right\rangle$ in the flavor basis are related to the charge radii $\left\langle r_{\nu_{j k}}^{2}\right\rangle$ in the mass basis by the relation [51]

$$
\left\langle r_{\nu_{\ell \ell^{\prime}}}^{2}\right\rangle=\sum_{j, k} U_{\ell j}^{*} U_{\ell^{\prime} k}\left\langle r_{\nu_{j k}}^{2}\right\rangle
$$

where $U$ is the neutrino mixing matrix. Therefore, even if the matrix of the neutrino charge radii is diagonal in the mass basis, transition charge radii are generated by the mixing.

The effects of the transition charge radii $\left\langle r_{\nu_{\ell \ell^{\prime}}}^{2}\right\rangle$, was discussed for the first time in Ref. [51] considering the case of elastic neutrino-electron scattering. Since the transition charge radii change the flavor of the neutrino in the final state of the elastic scattering process, the final state does not interfere with the weak interaction channel and the transition charge radii contributions add to the cross section incoherently with respect to the standard weak interaction contribution. In the case of coherent ${ }^{4}$ elastic neutrinonucleus scattering we consider the process

$$
\nu_{\ell}+\mathcal{N} \rightarrow \sum_{\ell^{\prime}} \nu_{\ell^{\prime}}+\mathcal{N}
$$

where $\mathcal{N}$ is the target nucleus. For a spin-zero nucleus and $T \ll E$, where $T$ is the nuclear kinetic recoil energy and $E$ is the neutrino energy, the differential cross section is given by

$$
\begin{aligned}
& \frac{d \sigma_{\nu_{\ell}-\mathcal{N}}}{d T}(E, T) \\
& \simeq \frac{G_{\mathrm{F}}^{2} M}{\pi}\left(1-\frac{M T}{2 E^{2}}\right)\left\{\left[\left(g_{V}^{p}-\tilde{Q}_{\ell \ell}\right) Z F_{Z}\left(|\vec{q}|^{2}\right)\right.\right. \\
& \left.\left.\quad+g_{V}^{n} N F_{N}\left(|\vec{q}|^{2}\right)\right]^{2}+Z^{2} F_{Z}^{2}\left(|\vec{q}|^{2}\right) \sum_{\ell^{\prime} \neq \ell}\left|\tilde{Q}_{\ell^{\prime} \ell}\right|^{2}\right\},
\end{aligned}
$$

\footnotetext{
${ }^{4}$ One should not confuse the meaning of the word "coherent" in "coherent elastic neutrino-nucleus scattering" with the coherency or incoherency of the charge radii contributions with respect to the standard weak interactions. The first coherency refers to the response of the nucleus as a whole to the interaction, whereas the second coherency refers to the interference of the final neutrino state.
}

with $^{5}$

$$
\begin{gathered}
g_{V}^{p}=\frac{1}{2}-2 \sin ^{2} \vartheta_{W}, \\
g_{V}^{n}=-\frac{1}{2}, \\
\tilde{Q}_{\ell \ell^{\prime}}=\frac{2}{3} m_{W}^{2} \sin ^{2} \vartheta_{W}\left\langle r_{\nu_{\ell \ell^{\prime}}}^{2}\right\rangle .
\end{gathered}
$$

In Eq. (14) one can distinguish the effects of the diagonal charge radii $\left\langle r_{\nu_{\ell}}^{2}\right\rangle \equiv\left\langle r_{\nu_{e}}^{2}\right\rangle$ that contribute through the addition of $\tilde{Q}_{\ell \ell}$ to $g_{V}^{p}$, which is equivalent to the shift in Eq. (1). This contribution affects only the protons in the nucleus, whose number is given by $Z$. On the other hand, the transition charge radii $\left\langle r_{\nu_{\ell \ell^{\prime}}}^{2}\right\rangle$ with $\ell \neq \ell^{\prime}$ contribute to the cross section trough an additional term proportional to $Z^{2}$. The neutrons, whose number is given by $N$, of course do not interact with the neutrino charge radii. Note also that the charge radii of antineutrinos are related to those of neutrinos by ${ }^{6}\left\langle r_{\bar{\nu}_{\ell \ell^{\prime}}}^{2}\right\rangle=-\left\langle r_{t^{\prime} \ell}^{2}\right\rangle$. This is important for the diagonal charge radii that contribute coherently with weak interactions in the cross section (14). Since

$$
\left\langle r_{\bar{\nu}_{\ell}}^{2}\right\rangle=-\left\langle r_{\nu_{\ell}}^{2}\right\rangle,
$$

neutrinos and antineutrinos contribute with different signs to the shift of $\sin ^{2} \vartheta_{W}$ in Eq. (1).

For the proton and neutron contributions in Eq. (14) we take into account the corresponding nuclear form factors $F_{Z}\left(|\vec{q}|^{2}\right)$ and $F_{N}\left(|\vec{q}|^{2}\right)$, which are the Fourier transforms of the corresponding nucleon distribution in the nucleus and describe the loss of coherence for $|\vec{q}| R \gtrsim 1$, where $R$ is the nuclear radius. These distributions are usually expressed with an appropriate parametrization which depends on two parameters: the rms radius $R$ and the surface thickness $s$. The most common parametrizations are the Fermi, symmetrized Fermi [60], and Helm [61]. Since these different parametrization are practically equivalent in the analysis of COHERENT data [33], for simplicity in the following we use only the Helm parametrization [61]

$$
F\left(|\vec{q}|^{2}\right)=3 \frac{j_{1}\left(|\vec{q}| R_{0}\right)}{|\vec{q}| R_{0}} e^{-|\vec{q}|^{2} s^{2} / 2}
$$

where $j_{1}(x)=\sin (x) / x^{2}-\cos (x) / x$ is the spherical Bessel function of order one and $R_{0}$ is related to the rms radius $R$ by

\footnotetext{
${ }^{5} \mathrm{We}$ neglect the radiative corrections to $g_{V}^{p}$ and $g_{V}^{n}$ (see Ref. [24]), that are too small to affect our results.

${ }^{6}$ From Eqs. (3.48) and (7.33) of Ref. [1], we have $\left\langle r_{\bar{L}_{j k}}^{2}\right\rangle=$ $-\left\langle r_{\nu_{k j}}^{2}\right\rangle$. Since $\left\langle r_{\bar{\nu}_{t \ell^{\prime}}}^{2}\right\rangle=\sum_{j, k} U_{\ell_{j}} U_{\ell^{\prime} k}^{*}\left\langle r_{\nu_{j k}}^{2}\right\rangle$, from Eq. (12) we obtain $\left\langle r_{\bar{\nu}_{t \epsilon^{\prime}}}^{2}\right\rangle=-\left\langle r_{\nu_{t^{\prime} \ell}}^{2}\right\rangle$.
} 


$$
R^{2}=\frac{3}{5} R_{0}^{2}+3 s^{2} .
$$

For the surface thickness $s$ we consider the value $s=$ $0.9 \mathrm{fm}$ that was determined for the proton form factor of similar nuclei [62].

The COHERENT experiment measured the coherent elastic scattering of neutrinos on ${ }^{133} \mathrm{Cs}$ and ${ }^{127} \mathrm{I}$. Hence, the total cross section is given by

$$
\frac{d \sigma_{\nu-\mathrm{CSI}}}{d T}=\frac{d \sigma_{\nu-\mathrm{Cs}}}{d T}+\frac{d \sigma_{\nu-\mathrm{I}}}{d T},
$$

with $N_{\mathrm{Cs}}=78, Z_{\mathrm{Cs}}=55, N_{\mathrm{I}}=74$, and $Z_{\mathrm{I}}=53$. We neglect the small axial contribution due to the unpaired valence protons [24].

In our analysis of the COHERENT data we use the values of the rms radii of the proton distribution of ${ }^{133} \mathrm{Cs}$ and ${ }^{127} \mathrm{I}$ that have been determined with high accuracy with muonic atom spectroscopy [63]:

$$
\begin{gathered}
R_{p}\left({ }^{133} \mathrm{Cs}\right)=4.804 \mathrm{fm}, \\
R_{p}\left({ }^{127} \mathrm{I}\right)=4.749 \mathrm{fm} .
\end{gathered}
$$

On the other hand, there is no experimental determination of the value of the rms radii of the neutron distribution of ${ }^{133} \mathrm{Cs}$ and ${ }^{127} \mathrm{I}$, except that obtained in Ref. [33] from the analysis of the COHERENT data assuming the absence of effects due to the neutrino charge radii and nonstandard interactions. Therefore, in order to extract information on the neutrino charge radii from the COHERENT data we adopt the following two approaches:

Fixed $R_{n}$ : We assume the theoretical values

$$
\begin{gathered}
R_{n}\left({ }^{133} \mathrm{Cs}\right)=5.01 \mathrm{fm}, \\
R_{n}\left({ }^{127} \mathrm{I}\right)=4.94 \mathrm{fm},
\end{gathered}
$$

obtained in the relativistic mean field (RMF) NL-Z2 [64] nuclear model calculated in Ref. [33]. This is a reasonable assumption taking into account that the values of the rms radii of the proton distribution of ${ }^{133} \mathrm{Cs}$ and ${ }^{127} \mathrm{I}$ calculated with the RMF NL-Z2, $R_{p}\left({ }^{133} \mathrm{Cs}\right)=4.79 \mathrm{fm}$ and $R_{p}\left({ }^{127} \mathrm{I}\right)=4.73 \mathrm{fm}$, are in approximate agreement with the experimental values in Eqs. (22) and (23).

Free $R_{n}$ : We perform a fit of the coherent data with free $R_{n}\left({ }^{133} \mathrm{Cs}\right)$ and $R_{n}\left({ }^{127} \mathrm{I}\right)$ which are allowed to vary in a suitable interval. For the lower bounds of the allowed ranges of $R_{n}\left({ }^{133} \mathrm{Cs}\right)$ and $R_{n}\left({ }^{127} \mathrm{I}\right)$ we took the corresponding experimental values of $R_{p}\left({ }^{133} \mathrm{Cs}\right)$ and $R_{p}\left({ }^{127} \mathrm{I}\right)$ in Eqs. (22) and (23). These are very reliable lower bounds, because in ${ }^{133} \mathrm{Cs}$ and ${ }^{127} \mathrm{I}$ there are about 20 more neutrons than protons and all model calculations predict $R_{n}>R_{p}$. The upper bounds for $R_{n}\left({ }^{133} \mathrm{Cs}\right)$ and $R_{n}\left({ }^{127} \mathrm{I}\right)$ are more arbitrary, since there is no experimental information. However, the parityviolating PREX experiment measured $R_{n}\left({ }^{208} \mathrm{~Pb}\right)=$ $5.75 \pm 0.18 \mathrm{fm}[65,66]$. Since it is very unlikely that $R_{n}\left({ }^{133} \mathrm{Cs}\right)$ and $R_{n}\left({ }^{127} \mathrm{I}\right)$ are larger than $R_{n}\left({ }^{208} \mathrm{~Pb}\right)$, we adopt the upper bound of $6 \mathrm{fm}$. In any case, we have checked that the limits that we obtain for the neutrino charge radii are stable within reasonable changes of the upper bounds for the allowed ranges of $R_{n}\left({ }^{133} \mathrm{Cs}\right)$ and $R_{n}\left({ }^{127} \mathrm{I}\right)$.

\section{FIT OF THE TIME-INTEGRATED COHERENT ENERGY SPECTRUM}

As a first step, we fitted the time-integrated COHERENT energy spectrum with the same method as in Ref. [33], using the precise information in the COHERENT data release [19]. We considered the least-squares function

$$
\begin{aligned}
\chi^{2}= & \sum_{i=4}^{15}\left(\frac{N_{i}^{\mathrm{exp}}-(1+\alpha) N_{i}^{\mathrm{th}}-(1+\beta) B_{i}}{\sigma_{i}}\right)^{2} \\
& +\left(\frac{\alpha}{\sigma_{\alpha}}\right)^{2}+\left(\frac{\beta}{\sigma_{\beta}}\right)^{2} .
\end{aligned}
$$

For each energy bin $i, N_{i}^{\text {exp }}$ and $N_{i}^{\text {th }}$ are, respectively, the experimental and theoretical number of events, $B_{i}$ is the estimated number of background events, and $\sigma_{i}$ is the statistical uncertainty. $\alpha$ and $\beta$ are nuisance parameters which quantify, respectively, the systematic uncertainty of the signal rate and the systematic uncertainty of the background rate with corresponding standard deviations $\sigma_{\alpha}=0.28$ and $\sigma_{\beta}=0.25$ [18]. The fit is restricted to the bin numbers from 4 to 15 where the acceptance function is nonzero and the linear relation $N_{\mathrm{PE}}=1.17 T / \mathrm{keV}$ between the observed number of photoelectrons $N_{\mathrm{PE}}$ and the nuclear kinetic recoil energy $T$ is reliable [18].

The theoretical number of coherent elastic scattering events $N_{i}^{\text {th }}$ in each energy bin $i$ depends on the neutrino charge radii and on the nuclear form factors. It is given by

$$
N_{i}^{\mathrm{th}}=N_{\mathrm{CsI}} \int_{T_{i}}^{T_{i+1}} d T \int_{E_{\min }} d E A(T) \frac{d N_{\nu}}{d E} \frac{d \sigma_{\nu-\mathrm{CsI}}}{d T},
$$

where $N_{\text {CSI }}$ is the number of CsI in the detector (given by $N_{\mathrm{A}} M_{\text {det }} / M_{\text {CsI }}$, where $N_{\mathrm{A}}$ is the Avogadro number, $M_{\mathrm{det}}=14.6 \mathrm{~kg}$, is the detector mass, and $M_{\mathrm{CsI}}=259.8$ is the molar mass of CsI), $E_{\min }=\sqrt{M T / 2}, A(T)$ is the acceptance function and $d N_{\nu} / d E$ is the neutrino flux integrated over the experiment lifetime. Neutrinos at the Spallation Neutron Source consist of a prompt component of monochromatic $\nu_{\mu}$ from stopped pion decays, $\pi^{+} \rightarrow \mu^{+}+\nu_{\mu}$, and two delayed components of $\bar{\nu}_{\mu}$ and 


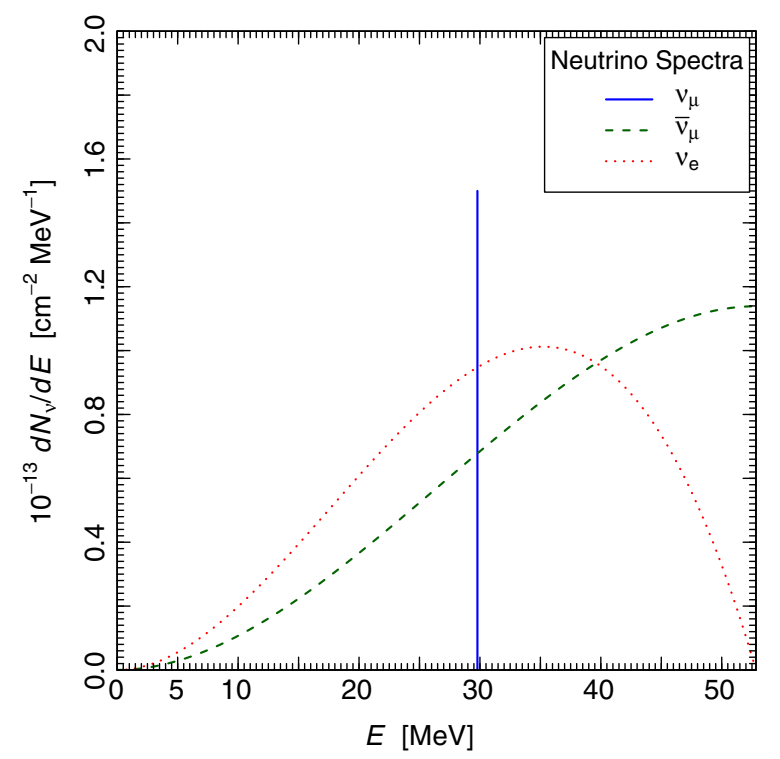

FIG. 1. The COHERENT $\nu_{\mu}, \bar{\nu}_{\mu}$, and $\nu_{e}$ spectra.

$\nu_{e}$ from the subsequent muon decays, $\mu^{+} \rightarrow e^{+}+\bar{\nu}_{\mu}+\nu_{e}$. The total flux $d N_{\nu} / d E$ is the sum of

$$
\begin{aligned}
& \frac{d N_{\nu_{\mu}}}{d E}=\eta \delta\left(E-\frac{m_{\pi}^{2}-m_{\mu}^{2}}{2 m_{\pi}}\right), \\
& \frac{d N_{\nu_{\bar{\mu}}}}{d E}=\eta \frac{64 E^{2}}{m_{\mu}^{3}}\left(\frac{3}{4}-\frac{E}{m_{\mu}}\right), \\
& \frac{d N_{\nu_{e}}}{d E}=\eta \frac{192 E^{2}}{m_{\mu}^{3}}\left(\frac{1}{2}-\frac{E}{m_{\mu}}\right),
\end{aligned}
$$

for $E \leq m_{\mu} / 2 \simeq 52.8 \mathrm{MeV}$, with the normalization factor $\eta=r N_{\mathrm{POT}} / 4 \pi L^{2}$, where $r=0.08$ is the number of neutrinos per flavor that are produced for each proton on target, $N_{\text {POT }}=1.76 \times 10^{23}$ is the number of proton on target and $L=19.3 \mathrm{~m}$ is the distance between the source and the COHERENT CsI detector [18]. The three neutrino spectra are illustrated in Fig. 1. Note that for the $\bar{\nu}_{\mu}$ spectrum it is important to take into account the relation in Eq. (18).

Figure 2 illustrates the effects of the neutrino charge radii $\left\langle r_{\nu_{e}}^{2}\right\rangle$ and $\left\langle r_{\nu_{\mu}}^{2}\right\rangle$ in the fit of the COHERENT data. Note that for $\left|\left\langle r_{\nu_{e, \mu}}^{2}\right\rangle\right| \simeq 20 \times 10^{-32} \mathrm{~cm}^{2}$ the contribution of the proton term in Eq. (14) becomes similar to that of the neutron term, which is dominant in the absence of neutrino charge radii. Therefore, since the uncertainties of the COHERENT data are quite large, we expect to obtain limits on the neutrino charge radii of the order of $20 \times 10^{-32} \mathrm{~cm}^{2}$. Indeed, one can see from Fig. 2 that values of $\left\langle r_{\nu_{e}}^{2}\right\rangle$ and $\left\langle r_{\nu_{\mu}}^{2}\right\rangle$ of this size generate histograms that fit badly the data.

The first two columns in Table II and Fig. 3(a) show the results of the fit of the time-integrated COHERENT energy spectrum considering only the effects of the diagonal neutrino charge radii $\left\langle r_{\nu_{e}}^{2}\right\rangle$ and $\left\langle r_{\nu_{\mu}}^{2}\right\rangle$. This restriction is appropriate for the measurement of the neutrino charge radii predicted by the standard model [Eqs. (5) and (6)], where there are no transition charge radii. From Table II one can see that the fits of the data are very good both with fixed and free $R_{n}$. The allowed ranges of $\left\langle r_{\nu_{e}}^{2}\right\rangle$ and $\left\langle r_{\nu_{\mu}}^{2}\right\rangle$ are some tens of $10^{-32} \mathrm{~cm}^{2}$, as expected. From Table II and Fig. 3(a) one can see that the allowed range of $\left\langle r_{\nu_{e}}^{2}\right\rangle$ is almost insensitive to the value of $R_{n}$, whereas the allowed range of $\left\langle r_{\nu_{\mu}}^{2}\right\rangle$ increases by about $30 \%$ in the free $R_{n}$ approach.

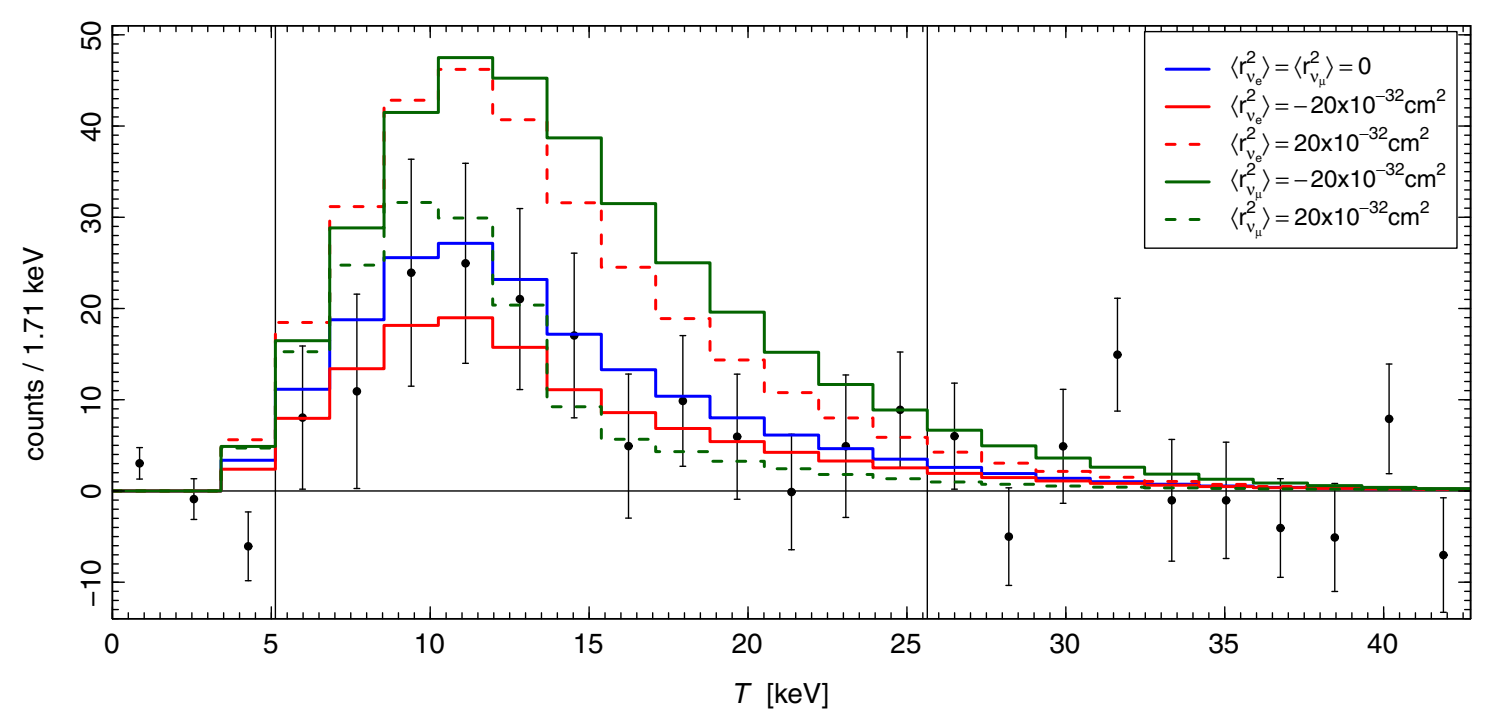

FIG. 2. COHERENT data [18,19] versus the nuclear kinetic recoil energy $T$. The blue histogram shows the theoretical predictions without neutrino charge radii and the theoretical values of the rms radii of the neutron distributions in Eqs. (24) and (25). The red and green histograms show the theoretical predictions obtained by adding the contributions of the neutrino charge radii reported in the legend. The two vertical lines at 5.13 and $25.64 \mathrm{keV}$ indicate the range of our analysis, as explained after Eq. (26). 


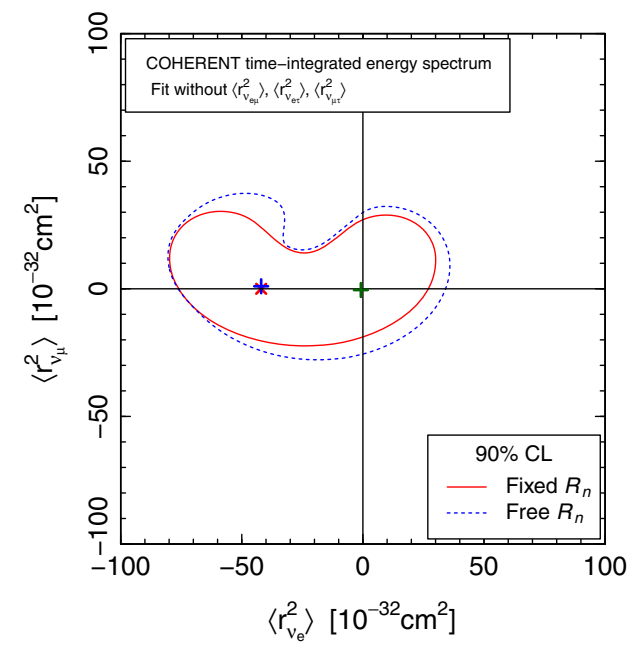

(a)

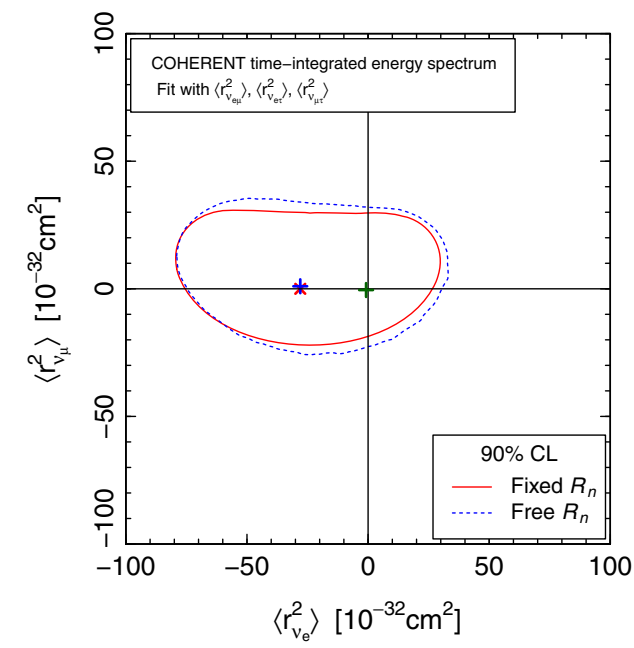

(b)

FIG. 3. $90 \% \mathrm{CL}$ allowed regions in the $\left\langle r_{\nu_{e}}^{2}\right\rangle-\left\langle r_{\nu_{\mu}}^{2}\right\rangle$ plane obtained from the fit of the time-integrated COHERENT energy spectrum without (a) and with (b) the transition charge radii. The red and blue points indicate the best-fit values. The green point near the origin indicates the standard model values in Eqs. (5) and (6).

The authors of Ref. [17] obtained bounds on $\left\langle r_{\nu_{e}}^{2}\right\rangle$ and $\left\langle r_{\nu_{\mu}}^{2}\right\rangle$ from an analysis of the total number of COHERENT events. Their results are different from ours, after taking into account the factor-of-two different definitions of the neutrino charge radii discussed in Sec. II. It is likely that this is due to the assumption in Ref. [17] of $\left\langle r_{\bar{\nu}_{\ell}}^{2}\right\rangle=\left\langle r_{\nu_{\ell}}^{2}\right\rangle$, contrary to the correct relation in Eq. (18). Indeed, we checked that making that assumption the fit of the total number of COHERENT events yields results similar to those presented in Ref. [17].

Let us now consider the fit of the time-integrated COHERENT energy spectrum in the complete theory including the effects of possible neutrino transition charge radii. The third and fourth columns in Table II and Fig. 3(b) show the results of the fits with fixed and free $R_{n}$. The marginal allowed ranges of $\left\langle r_{\nu_{e}}^{2}\right\rangle$ and $\left\langle r_{\nu_{\mu}}^{2}\right\rangle$ do not change significantly with respect to those obtained without the transition charge radii, although the contours in the $\left\langle r_{\nu_{e}}^{2}\right\rangle-\left\langle r_{\nu_{\mu}}^{2}\right\rangle$ plane change shape.

It is interesting that we obtained for the first time constraints on the neutrino transition charge radii. Their effect is illustrated in Fig. 4, where one can see that they always increase the predicted event rate, because their contribution adds incoherently to weak interactions in the cross section (14). From Table II and Fig. 3(b) one can also see that the limits on $\left\langle r_{\nu_{e}}^{2}\right\rangle$ and $\left\langle r_{\nu_{\mu}}^{2}\right\rangle$ are not sensitive to the assumed value of $R_{n}$, because the transition charge radii can compensate the effects of the variations of $R_{n}$.

Let us also note that the best-fit values of $\left\langle r_{\nu_{e}}^{2}\right\rangle-\left\langle r_{\nu_{\mu}}^{2}\right\rangle$ shown by points in Fig. 3 correspond to large negative values of $\left\langle r_{\nu_{e}}^{2}\right\rangle$ and very small values of $\left\langle r_{\nu_{\mu}}^{2}\right\rangle$. However, these indications do not have a sufficient statistical significance and it is wise to rely only on the 90\% CL contours in Fig. 3, which include the standard model values in Eqs. (5) and (6).

TABLE II. Results of the fits of the COHERENT data. The limits on the charge radii are at $90 \% \mathrm{CL}$ and in units of $10^{-32} \mathrm{~cm}^{2}$.

\begin{tabular}{|c|c|c|c|c|c|c|c|c|}
\hline & \multicolumn{4}{|c|}{ Spectrum } & \multicolumn{4}{|c|}{ Spectrum and time } \\
\hline & \multicolumn{2}{|c|}{$\left\langle r_{\nu_{e}}^{2}\right\rangle$ and $\left\langle r_{\nu_{\mu}}^{2}\right\rangle$ only } & \multicolumn{2}{|c|}{$\operatorname{All}\left\langle r_{\nu}^{2}\right\rangle$} & \multicolumn{2}{|c|}{$\left\langle r_{\nu_{e}}^{2}\right\rangle$ and $\left\langle r_{\nu_{\mu}}^{2}\right\rangle$ only } & \multicolumn{2}{|c|}{ All $\left\langle r_{\nu}^{2}\right\rangle$} \\
\hline & Fixed $R_{n}$ & Free $R_{n}$ & Fixed $R_{n}$ & Free $R_{n}$ & Fixed $R_{n}$ & Free $R_{n}$ & Fixed $R_{n}$ & Free $R_{n}$ \\
\hline$\chi_{\min }^{2}$ & 2.7 & 2.6 & 2.6 & 2.6 & 154.2 & 154.1 & 154.2 & 154.2 \\
\hline $\mathrm{NDF}$ & 10 & 8 & 7 & 5 & 142 & 140 & 139 & 137 \\
\hline GoF & $99 \%$ & $96 \%$ & $92 \%$ & $76 \%$ & $23 \%$ & $20 \%$ & $18 \%$ & $15 \%$ \\
\hline$\left\langle r_{\nu_{e}}^{2}\right\rangle$ & $(-69,19)$ & $(-70,25)$ & $(-69,19)$ & $(-69,23)$ & $(-63,12)$ & $(-63,13)$ & $(-63,12)$ & $(-63,12)$ \\
\hline$\left\langle r_{\nu_{\mu}}^{2}\right\rangle$ & $(-15,21)$ & $(-21,27)$ & $(-15,22)$ & $(-20,26)$ & $(-7,9)$ & $(-7,12)$ & $(-7,9)$ & $(-8,11)$ \\
\hline$\left|\left\langle r_{\nu_{e \mu}}^{2}\right\rangle\right|$ & & & $<25$ & $<27$ & & & $<22$ & $<22$ \\
\hline$\left|\left\langle r_{\nu_{e \tau}}^{2}\right\rangle\right|$ & & & $<44$ & $<46$ & & & $<37$ & $<38$ \\
\hline$\left|\left\langle r_{\nu_{\mu \tau}}^{2 \tau \tau}\right\rangle\right|$ & & & $<31$ & $<32$ & & & $<26$ & $<27$ \\
\hline
\end{tabular}




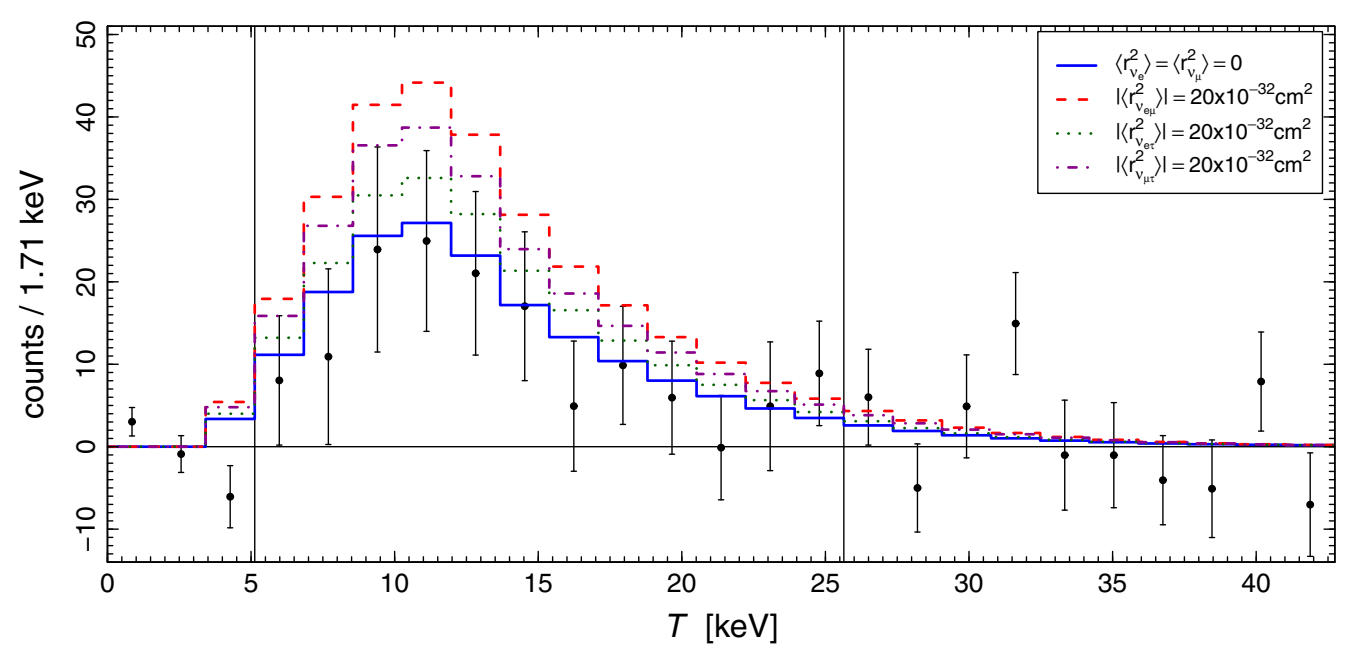

FIG. 4. COHERENT data [18,19] versus the nuclear kinetic recoil energy $T$. The blue histogram shows the theoretical predictions without neutrino charge radii and the theoretical values of the rms radii of the neutron distributions in Eqs. (24) and (25). The dashed red, dotted green and dash-dotted magenta histograms show the theoretical predictions obtained by adding the contributions of the neutrino transition charge radii reported in the legend. The two vertical lines at 5.13 and $25.64 \mathrm{keV}$ indicate the range of our analysis, as explained after Eq. (26).

\section{FIT OF THE TIME-DEPENDENT COHERENT DATA}

The data release in Ref. [19] of the COHERENT collaboration contains the complete information on the energy and arrival time of the observed events. The time of arrival after protons-on-targets is an important information for the study of neutrino properties, because $\nu_{\mu}$ 's produced by $\pi^{+}$decay arrive promptly within about $1.5 \mu \mathrm{s}$, whereas $\bar{\nu}_{\mu}$ 's and $\nu_{e}$ 's produced by $\mu^{+}$arrive in a time interval of about $10 \mu$ s (see Fig. 5). Hence, the time distribution of the

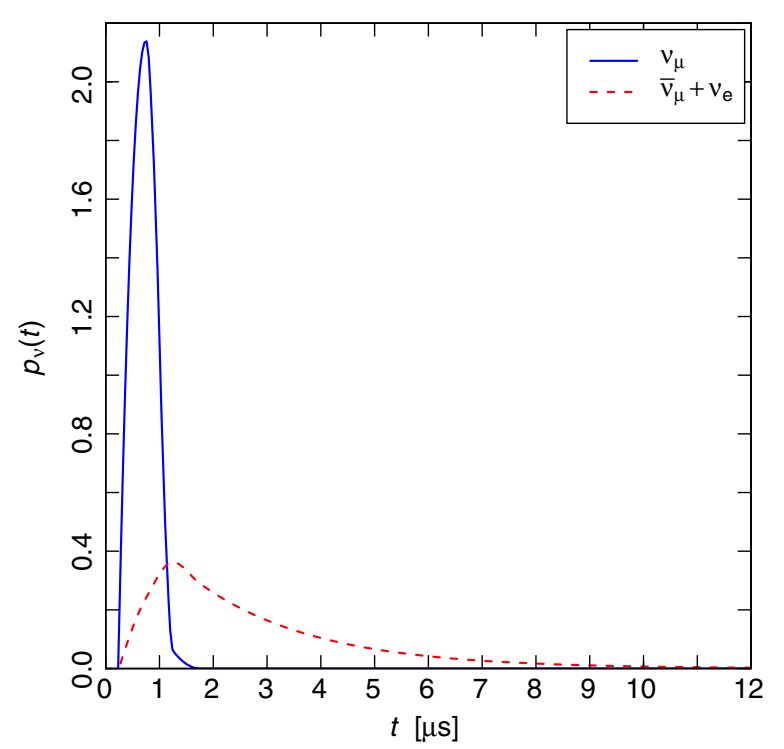

FIG. 5. The time distribution of arrivals after protons-on-targets of prompt $\nu_{\mu}$ 's and delayed $\bar{\nu}_{\mu}$ 's and $\nu_{e}$ 's in the COHERENT experiment $[18,19]$. data increases the information on the difference between the properties of $\nu_{\mu}$ and those of $\bar{\nu}_{\mu}$ and $\nu_{e}$.

We analyzed the time-dependent COHERENT data in the following way, according to the prescriptions given in the COHERENT data release [19]. Since in the binning of the events in energy and time there are some bins with zero count, we considered the Poissonian least-squares function [67]

$$
\begin{aligned}
\chi^{2}= & 2 \sum_{i=4}^{15} \sum_{j=1}^{12}\left[(1+\alpha) N_{i j}^{\mathrm{th}}+(1+\beta) B_{i j}\right. \\
& +(1+\gamma) N_{i j}^{\mathrm{bck}}-N_{i j}^{\mathrm{C}} \\
& \left.+N_{i j}^{\mathrm{C}} \ln \left(\frac{N_{i j}^{\mathrm{C}}}{(1+\alpha) N_{i j}^{\mathrm{th}}+(1+\beta) B_{i j}+(1+\gamma) N_{i j}^{\mathrm{bck}}}\right)\right] \\
& +\left(\frac{\alpha}{\sigma_{\alpha}}\right)^{2}+\left(\frac{\beta}{\sigma_{\beta}}\right)^{2}+\left(\frac{\gamma}{\sigma_{\gamma}}\right)^{2},
\end{aligned}
$$

where $i$ is the index of the energy bins, $j$ is the index of the time bins, $N_{i j}^{\text {th }}$ are the theoretical predictions that depend on the neutrino charge radii, $N_{i j}^{\mathrm{C}}$ are the coincidence (C) data, which contain signal and background events, $B_{i j}$ are the estimated neutron-induced backgrounds, $N_{i j}^{\mathrm{bck}}$ are the estimated backgrounds obtained from the anticoincidence (AC) data, $\sigma_{\alpha}=0.28$ is the systematic uncertainty of the signal rate, $\sigma_{\beta}=0.25$ is the systematic uncertainty of the neutroninduced background rate, and $\sigma_{\gamma}=0.05$ is the systematic uncertainty of the background estimated from the $\mathrm{AC}$ data $[18,19]$.

Table II and Fig. 6 show the results of the fit of the timedependent COHERENT data with fixed and free $R_{n}$ and without and with the neutrino transition charge radii. 


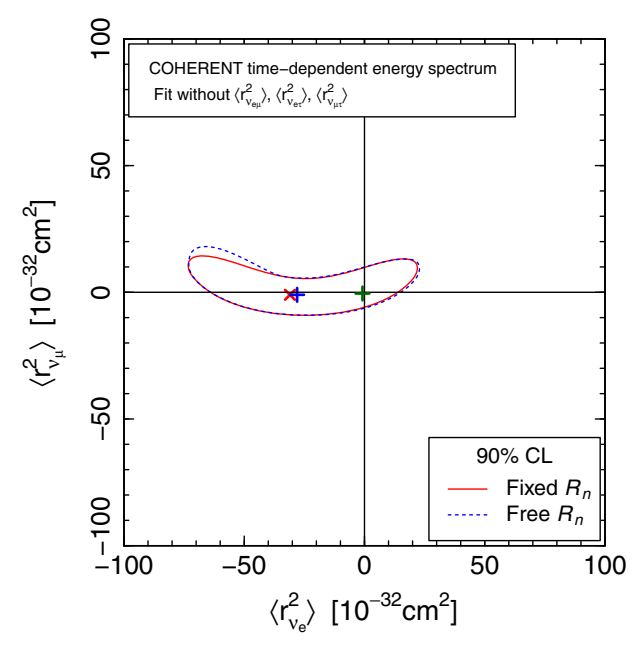

(a)

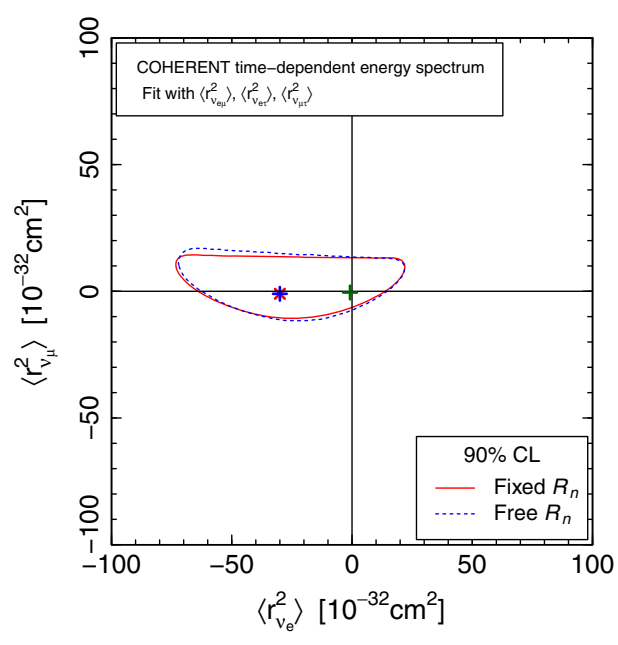

(b)

FIG. 6. 90\% CL allowed regions in the $\left\langle r_{\nu_{e}}^{2}\right\rangle-\left\langle r_{\nu_{\mu}}^{2}\right\rangle$ plane obtained from the fit of the time-dependent COHERENT energy spectrum without (a) and with (b) the transition charge radii. The red and blue points indicate the best-fit values. The green point near the origin indicates the standard model values in Eqs. (5) and (6).

Comparing these results with the corresponding allowed intervals in Table II and the corresponding allowed regions in Fig. 3 obtained from the fit of the time-integrated COHERENT data, one can see that, as expected, the time information allows us to obtain better constraints on the neutrino charge radii, especially $\left\langle r_{\nu_{\mu}}^{2}\right\rangle$. This is due to the difference of the prompt time distribution of $\nu_{\mu}$ 's and the delayed time distribution of $\bar{\nu}_{\mu}$ 's shown in Fig. 5, that severely constrains $\left\langle r_{\nu_{\mu}}^{2}\right\rangle$ through the relation (18). Therefore, the limits that we obtained $\left\langle r_{\nu_{\mu}}^{2}\right\rangle$ are comparable with those obtained in the BNL-E734 [47] and CHARM-II [48] experiments (see Table I).

The lower bound that we have obtained on $\left\langle r_{\nu_{e}}^{2}\right\rangle$ is about one order of magnitude less stringent than the experimental lower bounds in Table I, but the upper bound is comparable and confirms the results of those experiments.

One can also notice that the results on the neutrino charge radii are stable under variations of $R_{n}$, because the time dependence of the data is independent from the rms radii of the neutron distributions ${ }^{133} \mathrm{Cs}$ and ${ }^{127} \mathrm{I}$. Therefore, the limits obtained for the neutrino charge radii are independent from the nuclear model.

Moreover, from Table II and comparing Figs. 3(a) and 3(b), one can see that the inclusion in the analysis of the neutrino transition charge radii has little effect on the determination of $\left\langle r_{\nu_{e}}^{2}\right\rangle$ and $\left\langle r_{\nu_{\mu}}^{2}\right\rangle$. However, let us notice that the analysis of the time-dependent COHERENT data allows us to restrict the upper bounds on the neutrino transition charge radii obtained from the analysis of the time-integrated COHERENT data.

Let us finally note that, as in the case of the fit of the time-integrated COHERENT data commented at the end of Sec. III, the best-fit values of $\left\langle r_{\nu_{e}}^{2}\right\rangle-\left\langle r_{\nu_{\mu}}^{2}\right\rangle$ shown by points in
Fig. 6 correspond to large negative values of $\left\langle r_{\nu_{e}}^{2}\right\rangle$ and very small values of $\left\langle r_{\nu_{\mu}}^{2}\right\rangle$, but these indications do not have a sufficient statistical significance and we assert only the 90\% CL contours in Fig. 6, which include the standard model values in Eqs. (5) and (6).

\section{CONCLUSIONS}

Coherent elastic neutrino-nucleus scattering is a powerful tool to study neutrino and nuclear physics. In this paper we have analyzed the first data on coherent elastic neutrinonucleus scattering obtained in the COHERENT experiment $[18,19]$ in order to get information on the neutrino charge radii, which are predicted in the standard model. We obtained limits on the diagonal charge radii $\left\langle r_{\nu_{e}}^{2}\right\rangle$ and $\left\langle r_{\nu_{\mu}}^{2}\right\rangle$, and on the transition charge radii $\left\langle r_{\nu_{e \mu}}^{2}\right\rangle,\left\langle r_{\nu_{e \tau}}^{2}\right\rangle$, and $\left\langle r_{\nu_{\mu \tau}}^{2}\right\rangle$ from different analyses of the time-integrated COHERENT energy spectrum and the time-dependent COHERENT data taking into account the uncertainty of the neutron distributions in the ${ }^{133} \mathrm{Cs}$ and ${ }^{127} \mathrm{I}$ parametrized by the corresponding rms radii.

We have shown that the time information of the COHERENT data allows us to restrict the allowed ranges of the charge radii, especially that of $\left\langle r_{\nu_{\mu}}^{2}\right\rangle$, for which we obtained the $90 \% \mathrm{CL}$ allowed interval

$$
-8 \times 10^{-32}<\left\langle r_{\nu_{\mu}}^{2}\right\rangle<11 \times 10^{-32} \mathrm{~cm}^{2},
$$

marginalizing over reliable allowed intervals of the rms radii of the neutron distributions of ${ }^{133} \mathrm{Cs}$ and ${ }^{127} \mathrm{I}$. This limit is comparable with the BNL-E734 [47] and CHARM-II [48] limits in Table I.

We have obtained for the first time limits on the neutrino transition charge radii from experimental data (see 
Table II), taking into account their effect in the cross section according to Ref. [51]:

$\left(\left|\left\langle r_{\nu_{e \mu}}^{2}\right\rangle\right|,\left|\left\langle r_{\nu_{e \tau}}^{2}\right\rangle\right|,\left|\left\langle r_{\nu_{\mu \tau}}^{2}\right\rangle\right|\right)<(22,38,27) \times 10^{-32} \mathrm{~cm}^{2}$,

at $90 \% \mathrm{CL}$, marginalizing over reliable allowed intervals of the rms radii of the neutron distributions of ${ }^{133} \mathrm{Cs}$ and ${ }^{127} \mathrm{I}$. This is an interesting information on the physics beyond the standard model which can generate the neutrino transition charge radii [68].

The limits on the diagonal neutrino charge radii $\left\langle r_{\nu_{e}}^{2}\right\rangle$ and $\left\langle r_{\nu_{\mu}}^{2}\right\rangle$ that we have obtained are not better than the previous limits in Table I, but our analysis confirms those limits and hints at the likeliness of obtaining more stringent limits with the oncoming more precise data of the COHERENT experiment [69] and other coherent elastic neutrino-nucleus scattering experiments (CONUS [70], CONNIE [71], MINER [72], $\nu$-cleus [73], TEXONO [74], and others).

\section{ACKNOWLEDGMENTS}

We would like to thank Grayson Rich and Kate Scholberg for enlightening discussions on the COHERENT experiment. The work of Y. F. Li and Y. Y. Zhang is supported in part by the National Natural Science Foundation of China under Grant No. 11835013, by the Strategic Priority Research Program of the Chinese Academy of Sciences under Grant No. XDA10010100. The work of K. A. Kouzakov, Y. F. Li, Y. Y. Zhang, and A. I. Studenikin was supported in part by the joint project of the National Natural Science Foundation of China under Grant No. 1161101153, and the Russian Foundation for Basic Research under Grant No. 17-52-53133 GFEN_a. Y.F. Li is also grateful for the support by the CAS Center for Excellence in Particle Physics (CCEPP). K. A. Kouzakov and A. I. Studenikin are grateful for the support by the Russian Foundation for Basic Research under Grant No. 16-02-01023.
[1] C. Giunti and A. Studenikin, Rev. Mod. Phys. 87, 531 (2015).

[2] S. Y. Lee, Phys. Rev. D 6, 1701 (1972).

[3] B. W. Lee and R. E. Shrock, Phys. Rev. D 16, 1444 (1977).

[4] J. Lucio, A. Rosado, and A. Zepeda, Phys. Rev. D 29, 1539 (1984).

[5] J. Lucio, A. Rosado, and A. Zepeda, Phys. Rev. D 31, 1091 (1985).

[6] G. Degrassi, A. Sirlin, and W. J. Marciano, Phys. Rev. D 39, 287 (1989).

[7] J. Papavassiliou, Phys. Rev. D 41, 3179 (1990).

[8] J. Bernabeu, L. G. Cabral-Rosetti, J. Papavassiliou, and J. Vidal, Phys. Rev. D 62, 113012 (2000).

[9] J. Bernabeu, J. Papavassiliou, and J. Vidal, Phys. Rev. Lett. 89, 101802 (2002).

[10] J. Bernabeu, J. Papavassiliou, and J. Vidal, Nucl. Phys. B680, 450 (2004).

[11] K. Fujikawa and R. Shrock, Phys. Rev. D 69, 013007 (2004).

[12] J. Papavassiliou, J. Bernabeu, D. Binosi, and J. Vidal, Eur. Phys. J. C 33, S865 (2004).

[13] D. Binosi, J. Bernabeu, and J. Papavassiliou, Nucl. Phys. B716, 352 (2005).

[14] L. M. Sehgal, Phys. Lett. 162B, 370 (1985).

[15] J. Papavassiliou, J. Bernabeu, and M. Passera, Proc. Sci., HEP2005 (2006) 192 [arXiv:hep-ph/0512029].

[16] T. S. Kosmas, O. G. Miranda, D. K. Papoulias, M. Tortola, and J. W. F. Valle, Phys. Lett. B 750, 459 (2015).

[17] D. K. Papoulias and T. S. Kosmas, Phys. Rev. D 97, 033003 (2018).

[18] D. Akimov et al. (COHERENT Collaboration), Science 357, 1123 (2017).
[19] D. Akimov et al. (COHERENT Collaboration), arXiv:1804 .09459.

[20] D. Z. Freedman, Phys. Rev. D 9, 1389 (1974).

[21] D. Z. Freedman, D. N. Schramm, and D. L. Tubbs, Ann. Rev. Nucl. Part. Sci. 27, 167 (1977).

[22] A. Drukier and L. Stodolsky, Phys. Rev. D 30, 2295 (1984).

[23] V. A. Bednyakov and D. V. Naumov, Phys. Rev. D 98, 053004 (2018).

[24] J. Barranco, O. G. Miranda, and T. I. Rashba, J. High Energy Phys. 12 (2005) 021.

[25] K. Patton, J. Engel, G. C. McLaughlin, and N. Schunck, Phys. Rev. C 86, 024612 (2012).

[26] D. K. Papoulias and T. S. Kosmas, Adv. High Energy Phys. 2015, 763648 (2015).

[27] M. Lindner, W. Rodejohann, and X.-J. Xu, J. High Energy Phys. 03 (2017) 097.

[28] I. M. Shoemaker, Phys. Rev. D 95, 115028 (2017).

[29] E. Ciuffoli, J. Evslin, Q. Fu, and J. Tang, Phys. Rev. D 97, 113003 (2018).

[30] B. C. Canas, E. A. Garces, O. G. Miranda, and A. Parada, Phys. Lett. B 784, 159 (2018).

[31] J. Billard, J. Johnston, and B. J. Kavanagh, J. Cosmol. Astropart. Phys. 11 (2018) 016.

[32] V. Brdar, W. Rodejohann, and X.-J. Xu, arXiv:1810 03626.

[33] M. Cadeddu, C. Giunti, Y. Li, and Y. Zhang, Phys. Rev. Lett. 120, 072501 (2018).

[34] P. Coloma, M. C. Gonzalez-Garcia, M. Maltoni, and T. Schwetz, Phys. Rev. D 96, 115007 (2017).

[35] J. Liao and D. Marfatia, Phys. Lett. B 775, 54 (2017).

[36] P. B. Denton, Y. Farzan, and I. M. Shoemaker, J. High Energy Phys. 07 (2018) 037. 
[37] D. A. Sierra, V. De Romeri, and N. Rojas, Phys. Rev. D 98, 075018 (2018).

[38] M. Cadeddu and F. Dordei, arXiv:1808.10202.

[39] W. A. Bardeen, R. Gastmans, and B. Lautrup, Nucl. Phys. B46, 319 (1972).

[40] M. Dvornikov and A. Studenikin, Phys. Rev. D 69, 073001 (2004).

[41] M. Dvornikov and A. Studenikin, J. Exp. Theor. Phys. 99, 254 (2004).

[42] M. Tanabashi et al. (Particle Data Group), Phys. Rev. D 98, 030001 (2018).

[43] G. S. Vidyakin, V. N. Vyrodov, I. I. Gurevich, Y. V. Kozlov, V. P. Martemyanov, S. V. Sukhotin, V. G. Tarasenkov, E. V. Turbin, and S. K. Khakhimov, Pis'ma Zh. Eksp. Teor. Fiz. 55, 212 (1992) [JETP Lett. 55, 206 (1992)].

[44] M. Deniz et al. (TEXONO Collaboration), Phys. Rev. D 81, 072001 (2010).

[45] R. Allen, H. Chen, P. Doe, R. Hausammann, W. Lee et al., Phys. Rev. D 47, 11 (1993).

[46] L. B. Auerbach et al. (LSND Collaboration), Phys. Rev. D 63, 112001 (2001).

[47] L. Ahrens, S. Aronson, P. Connolly, B. Gibbard, M. Murtagh et al., Phys. Rev. D 41, 3297 (1990).

[48] P. Vilain et al. (CHARM-II Collaboration), Phys. Lett. B 345, 115 (1995).

[49] M. Hirsch, E. Nardi, and D. Restrepo, Phys. Rev. D 67, 033005 (2003).

[50] P. Vogel and J. Engel, Phys. Rev. D 39, 3378 (1989).

[51] K. A. Kouzakov and A. I. Studenikin, Phys. Rev. D 95, 055013 (2017).

[52] J. Barranco, O. G. Miranda, and T. I. Rashba, Phys. Lett. B 662, 431 (2008).

[53] A. Grau and J. Grifols, Phys. Lett. 166B, 233 (1986).

[54] S. Sakakibara and L. M. Sehgal, Phys. Lett. 83B, 77 (1979).

[55] J. Erler and S. Su, Prog. Part. Nucl. Phys. 71, 119 (2013).

[56] G. P. Zeller et al. (NuTeV Collaboration), Phys. Rev. Lett. 88, 091802 (2002).
[57] J. N. Bahcall, M. Kamionkowski, and A. Sirlin, Phys. Rev. D 51, 6146 (1995).

[58] S. Schael et al. (ALEPH, DELPHI, L3, OPAL, and SLD Collaborations, LEP Electroweak Working Group, SLD Electroweak Group, and SLD Heavy Flavour Group), Phys. Rep. 427, 257 (2006).

[59] K. S. McFarland et al. (CCFR Collaboration), Eur. Phys. J. C 1, 509 (1998).

[60] J. Piekarewicz, A. R. Linero, P. Giuliani, and E. Chicken, Phys. Rev. C 94, 034316 (2016).

[61] R. H. Helm, Phys. Rev. 104, 1466 (1956).

[62] J. Friedrich and N. Voegler, Nucl. Phys. A373, 192 (1982).

[63] G. Fricke, C. Bernhardt, K. Heilig, L. A. Schaller, L. Schellenberg, E. B. Shera, and C. W. de Jager, At. Data Nucl. Data Tables 60, 177 (1995).

[64] M. Bender, K. Rutz, P. G. Reinhard, J. A. Maruhn, and W. Greiner, Phys. Rev. C 60, 034304 (1999).

[65] S. Abrahamyan et al. (PREX Collaboration), Phys. Rev. Lett. 108, 112502 (2012).

[66] C. J. Horowitz et al., Phys. Rev. C 85, 032501 (2012).

[67] S. Baker and R. D. Cousins, Nucl. Instrum. Methods Phys. Res., Sect. A 221, 437 (1984).

[68] H. Novales-Sanchez, A. Rosado, V. Santiago-Olan, and J. Toscano, Phys. Rev. D 78, 073014 (2008).

[69] D. Akimov et al. (COHERENT Collaboration), arXiv:1803 .09183.

[70] W. Maneschg, "The Status of CONUS", Neutrino 2018, XXVIII International Conference on Neutrino Physics and Astrophysics, Heidelberg, Germany, 2018 (2018), DOI: 10.5281/zenodo. 1286927.

[71] A. Aguilar-Arevalo et al., J. Phys. Conf. Ser. 761, 012057 (2016); arXiv:1608.01565.

[72] G. Agnolet et al. (MINER Collaboration), Nucl. Instrum. Methods Phys. Res., Sect. A 853, 53 (2017).

[73] R. Strauss et al., Eur. Phys. J. C 77, 506 (2017).

[74] L. Singh and H. T. Wong (TEXONO Collaboration), J. Phys. Conf. Ser. 888, 012124 (2017). 